\title{
DISCUSSÃO SOBRE OS CRITÉRIOS DE AVALIAÇÃO ECONÔMICA: VALOR PRESENTE LÍQUIDO (VPL), VALOR ANUAL EQUIVALENTE (VAE) E VALOR ESPERADO DA TERRA $(\text { VET })^{1}$
}

\author{
Márcio Lopes da Silva² e Alessandro Albino Fontes ${ }^{3}$
}

\begin{abstract}
RESUMO - Na área florestal, com freqüência, têm ocorrido dúvidas e confusão na interpretação dos critérios de avaliação econômica. Este artigo objetivou esclarecer e discutir as principais questões relacionadas ao Valor Presente Líquido, Valor Anual Equivalente e Valor Esperado da Terra, bem como compará-los e demonstrar que há relação entre eles. Para tanto, utilizou-se um estudo de caso de um projeto de investimento em reflorestamento com eucalipto, a fim de comparar os critérios. Os resultados indicaram que todos os critérios são adequados e podem ser aplicados, bastando conhecer bem a diferença entre eles e a correta forma de interpretá-los.
\end{abstract}

Palavras-chave: Economia florestal, critérios de avaliação econômica e projeto florestal.

\section{DISCUSSION ON THE CRITERIA OF ECONOMICAL EVALUATION: NET PRESENT VALUE (VPL), EQUIVALENT ANNUAL VALUE (VAE) AND SOIL EXPECTED VALUE (VET)}

\begin{abstract}
Frequently, it has occurred doubts and confusion in the interpretation of criteria of economic evaluation in the forestry sector. The objective of this was to clarify and argue the main issues related to the Net Present Value, Equivalent Annual Value and Soil Expected Value, as well as to compare them and demonstrate that there is a relation among them. Therefore, a study of case of a project of investment in eucalyptus reforestation was used in order to compare the criteria. The results indicated that all the criteria were adjusted and could be applied, being enough to know well the difference among them and the correct form of their interpretation.
\end{abstract}

Keywords: Forestry economics, criteria of economic evaluation and forestry project.

\section{INTRODUÇÃ̃O}

A aplicação dos critérios de análise econômica na área florestal é fundamental para se decidir qual o melhor projeto e, ou, alternativa de manejo a serem adotados. Assim, a determinação da idade econômica de corte, o espaçamento, a adubação, a época e a intensidade de tratamentos silviculturais, e a espécie, dentre outras decisões, podem ser tomadas de forma mais segura quando feitas as simulações baseadas nos critérios técnico-econômicos (LOPES, 1990).
Muitos trabalhos têm sido feitos sobre análise econômica de projetos florestais, a maioria deles utilizando os principais critérios dessa análise econômica: Valor Presente Líquido (VPL), Taxa Interna de Retorno (TIR), Razão Benefício/Custo (B/C), Valor Anual Equivalente (VAE) e Custo Médio de Produção (CMP) (NAUTIYAL, 1988; SILVA et al., 1999; REZENDE e OLIVEIRA, 2001). Todos esses critérios levam em conta a variação do capital no tempo, mas cada um aponta diferentes aspectos relacionados aos projetos.

\footnotetext{
${ }^{1}$ Recebido em 05.10.2004 e aceito para publicação em 10.08.2005.

${ }^{2}$ Departamento de Engenharia Florestal da Universidade Federal de Viçosa. E-mail: <marlosil@ ufv.br>.

${ }^{3}$ Programa de Pós-Graduação em Ciência Florestal do DEF/UFV. E-mail: <aafontes@ alunos.ufv.br>.
} 
Outro critério muito aplicado é o Valor Esperado da Terra (VET), também conhecido como critério de Faustmann. Esse critério foi desenvolvido inicialmente pelo alemão Faustmann, para determinar o preço máximo de compra da terra nua a ser utilizada para determinada cultura, no caso a florestal. Como ele considerou o horizonte infinito, vem sendo amplamente aplicado também nas análises de projetos florestais e na seleção de alternativas de manejo florestal. Porém, freqüentemente surgem dúvidas e discussões sobre esses critérios, como: qual o melhor critério a ser adotado? Qual apresenta menos falhas? Qual deve ser utilizado para selecionar alternativas de manejo de diferentes durações? O VET é um critério de análise econômica ou não? Qual a relação entre VPL, VAE e VET? Quais as diferenças entre eles? Como interpretá-los?

Este artigo teve como objetivos discutir as principais questões relacionadas com o VPL, VAE e VET, esclarecer algumas dúvidas mais freqüentes e apontar a relação entre esses critérios de análise econômica de projetos florestais ou de seleção de alternativas de manejo florestal.

\section{MATERIAL E MÉTODOS}

\subsection{Critérios de Avaliação Econômica}

Foram utilizados os seguintes critérios de avaliação econômica: Valor Presente Líquido (VPL), Valor Anual Equivalente (VAE), Valor Presente Líquido Infinito (VPL $\infty$ ) e Valor Esperado da Terra (VET).

\subsubsection{VPL}

O valor presente líquido (VPL) de um projeto de investimento pode ser definido como a soma algébrica dos valores descontados do fluxo de caixa a ele associado. Em outras palavras, é a diferença do valor presente das receitas menos o valor presente dos custos. Assim:

$$
V P L=\sum_{j=0}^{n} R_{j}(1+i)^{-j}-\sum_{j=0}^{n} C_{j}(1+i)^{-j}
$$

em que:

$$
\begin{aligned}
& \mathrm{R}_{\mathrm{j}}=\text { valor atual das receitas; } \\
& \mathrm{C}_{\mathrm{j}}=\text { valor atual dos custos; } \\
& \mathrm{i}=\text { taxa de juros; } \\
& \mathrm{j}=\text { período em que as receitas ou os custos ocorrem; e } \\
& \mathrm{n}=\text { número de períodos ou duração do projeto. }
\end{aligned}
$$

O projeto que apresenta o VPL maior que zero (positivo) é economicamente viável, sendo considerado o melhor aquele que apresentar maior VPL. Para uso desse método, é necessária a definição de uma taxa de desconto (i).

\subsubsection{VAE}

OValor Anual Equivalente (VAE) é a parcela periódica e constante necessária ao pagamento de uma quantia igual ao VPL da opção de investimento em análise, ao longo de sua vida útil. Dito de outra forma, o VAE transforma o valor atual do projeto ou o seu VPL em fluxo de receitas ou custos periódicos e contínuos, equivalentes ao valor atual, durante a vida útil do projeto.

Para uma taxa de juros “ " $i$ ” unitária, relativa ao mesmo período que o adotado para o intervalo entre os fluxos de caixa, o VAE de um projeto pode ser calculado pela seguinte fórmula:

$$
V A E=\frac{V P L\left[(1+i)^{t}-1\right]}{1-(1+i)^{-n t}}
$$

em que:

$$
\begin{aligned}
& \mathrm{n}=\text { duração do projeto; e } \\
& \mathrm{t}=\text { número de períodos de capitalização; os demais }
\end{aligned}
$$
termos são conforme definidos.

Como o próprio nome indica, Valor "Anual" Equivalente pressupõe-se $\mathrm{t}=1$; $\operatorname{logo}$ a fórmula do VAE fica:

$$
V A E=\frac{V P L * i}{\left[1-(1+i)^{-n}\right]}
$$

O projeto será considerado economicamente viável se apresentar VAE positivo, indicando que os benefícios periódicos são maiores que os custos periódicos. Quanto à seleção de opções, deve ser escolhida a que apresentar maior VAE, para determinada taxa de desconto (REZENDE e OLIVEIRA, 2001).

\subsubsection{VET}

O método de Faustmann, comumente chamado de valor de expectativa da terra, ou valor esperado da terra (VET), ou valor de expectativa do solo, ou valor esperado do solo (VES), tem sido freqüentemente citado na literatura florestal.

O VET é um termo florestal usado para representar o valor presente líquido de uma área de terra nua a ser utilizada para a produção de madeira, calculado 
com base numa série infinita de rotações. Tal critério é mundialmente conhecido e utilizado para determinar a rotação econômica e o preço máximo de compra de terra nua, considerando-se uma série infinita, bem como para selecionar projetos alternativos (SILVA et al., 2002).

O cálculo do VET baseia-se na receita líquida perpétua (RT - CT), excluindo-se o custo da terra, a ser obtido de uma cultura (reflorestamento) e dado pela fórmula:

$$
V E T=\frac{V_{0} R L(1+i)^{t}}{(1+i)^{t}-1}
$$

em que:

$\mathrm{V}_{0} \mathrm{RL}=$ valor atual da receita líquida que se repete a cada ciclo os demais termos são conforme definidos.

O projeto será considerado economicamente viável se apresentar VET maior que o valor da terra.

O VET, por considerar o horizonte infinito, é amplamente utilizado na análise econômica de projetos florestais, pois elimina o problema de se compararem projetos com diferentes durações.

\subsubsection{VPL $\infty$}

O valor presente líquido calculado considerando a replicação do projeto para um horizonte infinito (VPL $\infty$ ) é dado pela fórmula:

$$
V P L \infty=\frac{V P L(1+i)^{t}}{(1+i)^{t}-1}
$$

em que os termos são conforme definidos.

À semelhança do VPL, o projeto que apresenta o VPL $\infty$ maior que zero (positivo) é economicamente viável, sendo considerado o melhor aquele que apresentar maior VPL $\infty$. Para o uso desse método, tambémé necessária a definição de uma taxa de desconto (i).

\subsection{Custo Anual da Terra}

O Custo Anual da Terra (CAT) será estimado ao considerar os juros (i) sobre o valor da terra (VT), conforme a seguinte fórmula:

$$
\mathrm{CAT}=\mathrm{VT} * \mathrm{i}
$$

\subsection{Volume Equivalente de Madeira}

O volume equivalente de madeira (VEM) será estimado, descontando-se a produção de madeira (PM) obtida na colheita, em dado período do tempo (n), pela taxa de juros (i), conforme a seguinte fórmula:

$$
V E M=\frac{P M}{(1+i)^{n}}
$$

\subsection{Estudo de Caso}

Para o estudo de caso, considerou-se o projeto de investimento em reflorestamento com eucalipto apresentado no Quadro 1.

\section{RESULTADOS E DISCUSSÃO}

\subsection{Fluxo de Caixa}

Com base nos valores do Quadro 1 e incluindo o custo anual da terra $(\mathrm{CAT}=400,00 * 0,10=40,00)$, temse o fluxo de caixa do projeto de investimento em reflorestamento com eucalipto (Figura 1).

\subsection{Critérios de Avaliação Econômica}

Calculou-se o valor presente dos custos do projeto de investimento em reflorestamento com eucalipto, sendo obtido:

$V_{0} C T=650+\frac{120}{1,1}+\frac{90}{(1,1)^{2}}+\frac{750}{(1,1)^{7}}+\frac{80\left[1-(1,1)^{-7}\right]}{0,1}+\frac{40\left[1-(1,1)^{-7}\right]}{0,1} \Rightarrow$

$\Rightarrow V_{0} C T=U S \$ 1.802,55 / h a$

Antes de calcular o valor presente das receitas do projeto, fez-se necessário o cálculo do volume equivalente de madeira, sendo obtido:

$$
V E M=\frac{250}{(1,1)^{7}}=128,29 \mathrm{~m}^{3} / \mathrm{ha}
$$

Quadro 1 - Projeto de investimento em reflorestamento com eucalipto

Table 1 - Investment project for reforestation with eucalyptus

\begin{tabular}{lcc}
\hline Itens & Ano de Ocorrência & Valor (US\$/ha) \\
\hline Custo de implantação & 0 & 650,00 \\
Custo de tratos culturais & 1 & 120,00 \\
Custo de tratos culturais & 2 & 90,00 \\
Custo anual* & $1-7$ & 80,00 \\
Valor da Terra & - & 400,00 \\
Colheita & 7 & $3,00 / \mathrm{m}^{3}$ \\
Produção (único corte) & 7 & $250 \mathrm{~m}^{3} / \mathrm{ha}^{3}$ \\
Preço da madeira & 7 & $18,00 / \mathrm{m}^{3}$ \\
Taxa de juros & - & $10 \%$ ao ano \\
\hline
\end{tabular}

* Gastos com combate à formiga, manutenção e administração. Fonte: Organizado pelos autores, em outubro de 2004. 


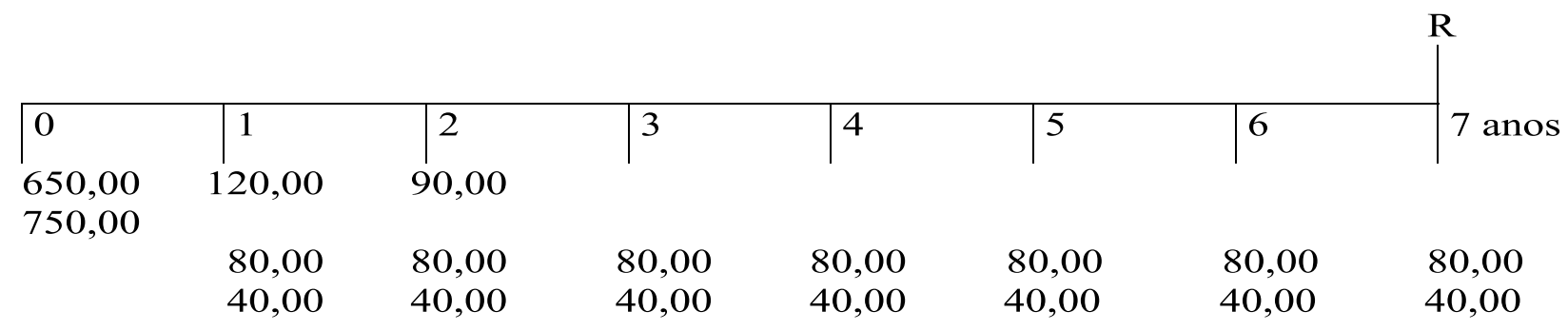

Fonte: Organizado pelos autores

Figura 1 - Fluxo de caixa do projeto de investimento em reflorestamento com eucalipto.

Figure 1 - Cash flow of the investment project for reforestation with eucalyptus.

Assim, o valor presente das receitas do projeto de investimento em reflorestamento com eucalipto obtido foi: $V_{0} R T=18 \times 128,29 \Rightarrow V_{0} R T=U S \$ 2.309,21 / h a$

\subsubsection{Cálculo do VPL}

Calculados os valores presentes dos custos e da receita, passou-se ao cálculo do valor presente líquido do projeto de investimento em reflorestamento com eucalipto:

$$
\begin{aligned}
\text { VPL } & =2.309,21-1.802,55 \\
\text { VPL } & =\text { US } \$ 506,66 / \text { ha }
\end{aligned}
$$

O VPL representa o lucro líquido e descontado para um horizonte de sete anos. Seu valor positivo indica que o projeto de investimento em questão é economicamente viável.

\subsubsection{Cálculo do VAE}

Após a obtenção do VPL, passou-se ao cálculo do valor anual equivalente do projeto de investimento em reflorestamento com eucalipto:

$$
\begin{aligned}
V A E & =\frac{506,66 \times 0,1}{1-(1,1)^{-7}} \\
\mathrm{VAE} & =\mathrm{US} \$ 104,07 / \mathrm{ha} / \mathrm{ano}
\end{aligned}
$$

Esse valor positivo para o VAE também indica que o projeto de investimento em questão é economicamente viável. O VAE representa o lucro descontado que o projeto proporciona a cada ano.

\subsubsection{Cálculo do VPL $\infty$}

Calculado o VPL, procedeu-se ao cálculo do valor presente líquido infinito do projeto de investimento em reflorestamento com eucalipto:

$$
V P L \infty=\frac{506,66(1+0,1)^{7}}{(1+0,1)^{7}-1}
$$

R. Árvore, Viçosa-MG, v.29, n.6, p.931-936, 2005
VPL $\infty=\mathrm{US} \$ 1.040,71 / \mathrm{ha}$

O VPL $\infty$ também apresentou valor positivo, indicando que o projeto de investimento em reflorestamento com eucalipto é economicamente viável.

\subsubsection{Relação entre VPL $\infty$ e VAE}

Partindo da fórmula do VAE (equação 3) e isolando o VPL, tem-se:

$$
V P L=\frac{V A E\left[1-(1+i)^{-n}\right]}{i}
$$

em que: $\mathrm{n}=\mathrm{t}=$ rotação.

Substituindo a fórmula (7) na fórmula do VPL $\infty$ (equação 5), tem-se:

$$
\begin{aligned}
& V P L_{\infty}=\frac{V A E\left[1-(1+i)^{-t}\right]}{i} \frac{(1+i)^{t}}{(1+i)^{t}-1} \\
& V P L_{\infty}=\frac{V A E\left[(1+i)^{t}-1\right]}{i\left[(1+i)^{t}-1\right]}
\end{aligned}
$$

Então,

$$
V P L_{\infty}=\frac{V A E}{i}
$$

ou, ainda,

$$
\mathrm{VAE}=\mathrm{VPL} \infty \mathrm{x} \text { i }
$$

Assim,

$$
\mathrm{VAE}=1.040,71 \times 0,1 \mathrm{P} \mathrm{VAE}=\mathrm{US} \$ 104,07 / \mathrm{ha} / \mathrm{ano} .
$$

Logo, pode-se dizer que tanto o VAE quanto o VPL $\infty$ conduzem ao mesmo resultado, portanto são mais adequados para comparar projetos com durações diferentes. 


\subsubsection{Cálculo do VET}

Este critério de avaliação econômica possui uma premissa, que é a exclusão do custo anual da terra dos cálculos. Assim, faz-se, novamente, necessário o cálculo do valor presente dos custos:

$$
\begin{aligned}
& V_{0} C T=650+\frac{120}{1,1}+\frac{90}{(1,1)^{2}}+\frac{750}{(1,1)^{7}}+\frac{80\left[1-(1,1)^{-7}\right]}{0,1} \Rightarrow \\
& \Rightarrow V_{0} C T=U S \$ 1.607,81 / h a
\end{aligned}
$$

O valor presente das receitas do projeto de investimento em reflorestamento com eucalipto não se altera, sendo:

$$
\mathrm{V}_{0} \mathrm{RT}=\mathrm{US} \$ 2.309,21 / \mathrm{ha}
$$

Então, tem-se o valor presente da receita líquida:

$\mathrm{V}_{0}$ Receita Líquida $=2.309,21-1.607,81=\mathrm{US} \$ 701,40 / \mathrm{ha}$.

Repetindo infinitamente essa receita a cada sete anos, tem-se:

$$
\begin{aligned}
V E T & =\frac{701,40(1+0,1)^{7}}{(1+0,1)^{7}-1} \\
V E T & =\mathrm{US} \$ 1.440,71 / \mathrm{ha}
\end{aligned}
$$

Esse valor encontrado para o VET é superior ao de mercado da terra (VT=US $\$ 400,00 /$ hectare), indicando que o projeto de investimento em reflorestamento com eucalipto é economicamente viável.

O VET indica o valor máximo que deve ser pago pela terra para a atividade em questão.

\subsubsection{Relação entre VET e VPL $\infty$}

A diferença entre o VET e o VPL $\infty$ é que o primeiro não considera o custo anual da terra (CAT), por isso seu valor é sempre maior. O custo anual da terra (CAT) pode ser dado por:

$$
\mathrm{CAT}=\mathrm{VT} * \mathrm{i} \Rightarrow V T=\frac{C A T}{i}
$$

Por isso, somando ao VET o valor atual do custo da terra (VT), que deve representar o seu valor de mercado, pode-se obter o VPL $\infty$. Assim:

$$
V E T=V P l_{\infty}+\frac{C A T}{i} \Rightarrow \mathrm{VET}=\mathrm{VPL} \infty+\mathrm{VT}
$$

Portanto, a relação entre o VET e o VPL $\infty$ pode ser expressa pela equação:

$$
\mathrm{VET}=\mathrm{VPL} \infty+\mathrm{VT} \quad(10)
$$

Assim, a diferença entre ambos é exatamente o valor da terra:

$$
\mathrm{VET}=1.040,71+400,00=\mathrm{US} \$ 1.440,71 / \mathrm{ha} .
$$

Logo, pode-se dizer que o VET representa o valor produtivo da terra ou o preço máximo que se pode pagar pela terra nua para determinada atividade econômica.

\subsubsection{Comparação entre os critérios}

No Quadro 2, apresenta-se um resumo comparativo entre os critérios de avaliação econômica abordados, no qual se observa que os critérios de avaliação econômica são bem diferentes. São conceitos distintos,

\begin{tabular}{|c|c|c|c|}
\hline Critério & Fórmula & Fórmula Alternativa & Valor (US\$/ha) \\
\hline VPL & $\sum R_{j}(1+i)^{-j}-\sum C_{j}(1+i)^{-j}$ & & 506,66 \\
\hline VAE & $\frac{V P L^{*} i}{1-\left[1-(1+i)^{-n}\right]}$ & $\mathrm{VPL} \infty * \mathrm{i}$ & 104,07 \\
\hline VPL $\infty$ & $\frac{V P L(1+i)^{t}}{(1+i)^{t}-1}$ & & $1.040,71$ \\
\hline VET & $\frac{V_{0} R L(1+i)^{t}}{(1+i)^{t}-1}$ & VPL $\infty+V T$ & $1.440,71$ \\
\hline
\end{tabular}
embora haja uma relação entre eles. Os valores obtidos também diferem muito. Caso o administrador não esteja ciente dessas diferenças entre os métodos ou não saiba como interpretá-los, isso pode levá-lo a tomar decisão ou escolher um projeto e, ou, alternativa de investimento que não seriam mais adequados.

Quadro 2 - Comparação entre os critérios de avaliação econômica Table 2 - Comparison among the criteria of economical evaluation

Fonte: Organizado pelos autores. 


\section{CONCLUSÕES}

Como principais conclusões deste estudo, citam-se:

- Tanto o VAE quanto o VPL $\infty$ conduzem ao mesmo resultado, portanto são mais adequados para comparar projetos com durações diferentes.

O VET pode ser utilizado para comparar alternativas de investimento, porém se deve ressaltar que, para um projeto ser viável por esse critério, o seu valor deve ser maior que o de mercado da terra e não maior que zero. Caso o VET seja menor que o valor da terra, o empreendimento é economicamente inviável.

- Há diferença entre o VPL $\infty$ e o VET. São conceitos diferentes, e, quando se computa o custo da terra no cálculo, o que se obtém é o VPL $\infty$ e não o VET, por isso é comum encontrar trabalhos errados, pois se trocam os conceitos.

\section{REFERÊNCIAS BIBLIOGRÁFICAS}

LOPES, H.V.S. Análise econômica dos fatores que afetam a rotação de povoamentos de eucaliptos. 1990. 188f. Dissertação (Mestrado em Ciência Florestal) - Universidade Federal de Viçosa, Viçosa, 1990.

NAUTIYAL, J.C. Forest economics. Principles and applications. Toronto: Canadian Scholars' Press, 1988. 851p.

REZENDE, J.L.P.; OLIVEIRA, A.D. Análise econômica e social de projetos florestais. Viçosa: Universidade Federal Viçosa, 2001.389p.

SILVA, M.L.;JACOVINE,L.A.G; VALVERDE, S.R. Economia florestal. Viçosa: Universidade Federal Viçosa, 2002. 178p.

SILVA, M.L.; FONTES, A.A.; LEITE, H.G. Rotação econômica em plantações de eucalipto nãodesbastadas e destinadas a multiprodutos. Revista Árvore, v.23, n.4, p.403-412, 1999. 